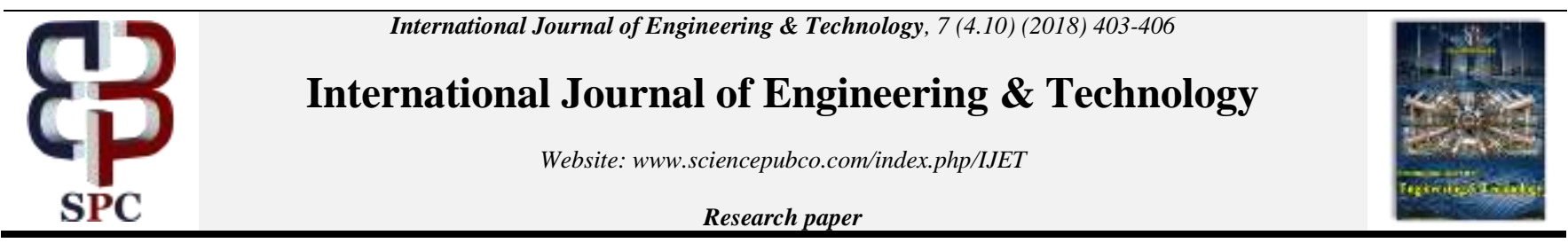

\title{
Edge Version Molecular Descriptors of Tetrameric 1, 3 Adamantane
}

\author{
G. Mohanappriya ${ }^{1 *}$, D. Vijiyalakshmi ${ }^{2}$ \\ 1,2 Department of Mathematics, Kongunadu Arts and Science College, \\ Coimbatore-641029, Tamil Nadu, India. \\ *Corresponding author E-mail: mohanappriyag25@gmail.com
}

\begin{abstract}
Molecular descriptors (Topological indices) are the numerical invariants of a molecular graph which distinguish its topology. In this article, we compute edge version of topological indices such as Zagreb index, Atom bond connectivity index, Fourth atom bond connectivity index, Geometric Arithmetic index and Fifth Geometric Arithmetic index of tetrameric 1,3 adamantane.
\end{abstract}

Keywords: Molecular Descriptors, Degree based invariants, Line Graph, Tetrameric 1; 3 Adamantane.

\section{Introduction}

Chemical Graph Theory devotes graph concepts to encode the structure of a molecule. A chemical graph cites to a mathematical object that symbolizes the structure of several interconnections of a molecule. A chemical graph is basically a statement about nodes and links and their relations. A key concept in chemical graph theory and in molecular connectivity is the concept of valence of a vertex encoding atom, i.e., its connectivity degree. For a molecular graph $\mathrm{H}$, with the vertex set $\mathrm{V}(\mathrm{H})$ and edge set $\mathrm{E}(\mathrm{H})$, a topological index is a numeric measure that can be computed from $\mathrm{H}$. The order of a graph is $|V(H)|$, represents cardinality of vertex (node) set denoted by $m$. The size of a graph is $|\mathrm{E}(\mathrm{H})|$, represents cardinality of edge (link) set denoted by $n$. The degree (valency) of a vertex $\mathrm{v}$ is the number of edges incident to $\mathrm{v}$ denoted by $\operatorname{deg}(\mathrm{v})$. $\mathrm{s}_{\mathrm{v}}=\Sigma_{\mathrm{u} \in \mathrm{Nv}} \operatorname{deg}(\mathrm{v})$, where $\mathrm{N}_{\mathrm{v}}=\{\mathrm{u} \in \mathrm{V}(\mathrm{H})$ : $\mathrm{uv} \in \mathrm{E}(\mathrm{H})\}$. The Line graph $\mathrm{L}(\mathrm{H})$ of a graph is the simple graph in such a way that the links in $\mathrm{H}$ are replaced by nodes in $\mathrm{L}(\mathrm{H})$ and two nodes in $\mathrm{L}(\mathrm{H})$ are connected whenever the corresponding links in $\mathrm{H}$ are adjacent. Comparing to other Molecular Building Blocks (MBBs) diamonds are most significant allotrope of carbon for its unique structure and chemical properties. Diamondoids are widely used in construction of nanostructures, a well known MBBs is diamondoids is shown in figure 1. The basic repeated unit of diamondoids is adamantane. Applications of diamondoids in the field of petroleum science, geochemistry, biomedicine, material science and nanotechnology can be found in $[2,3,4,11]$.

Molecular descriptors such as generalized Randic index, Zagreb index, general sum connectivity index, atom bond connectivity index and geometric arithmetic index are taken in account for the analyze of bio-activity of a chemical compound in the study of QSAR/QSPR. For more results on molecular descriptors and edge version of nanostructures reader can refer $[7,8,9,10]$.

The study of topological indices was effectively initiated in 1947 in chemistry by Wiener. He introduced a distance based topological indices called Wiener index[13].

\section{Edge version of topological indices}

The edge version of General Randic index was introduced by Bollobas and Erdos [5] defined as

$$
e R_{u(u)}(\mathrm{H})=\sum_{u v \in \mathbb{E}(L(H))}\left(d_{U L H)}(u) d_{L U H)}(\mathrm{v})\right)^{\alpha}
$$

for $\alpha=1,1 / 2,-1 / 2,1$ and where $d_{L(H)}(u)$ is the valency of the node $e$ $d_{L(H)}(v)$ is the valency of the node of line graph $H$.

In [7], Gutman and Trinajistic defined the edge version of Zagreb index as

$e M_{1}(\mathrm{H})=\sum_{u v \in E(L(H))}\left(d_{L(H)}(u)+d_{L(H)}(\mathrm{v})\right)$

where $d_{L(H)}(u)$ is the valency of the node $u$ and $d_{L(H)}(v)$ is the valency of the node $v$ of line graph $H$.

Estrada et al reported the Atom Bond Connectivity index [5] and its edge version is defined by

$$
{ }_{e} A B C(H)=\sum_{u v \in E(L(G))} \sqrt{\frac{d_{L(H)}(u)+d_{L(H)}(v)-2}{d_{L(H)}(u) d_{L(H)}(v)}}
$$

where $d_{L(H)}(u)$ is the valency of the node $e$ and $d_{L(H)}(v)$ is the valency of the node $v$ of line graph $H$.

The fourth edge version of Atom Bond Connectivity index is defined as

$$
e A B C_{4}(\mathrm{H})=\sum_{w \in E(L(H))} \sqrt{\frac{\left(s_{L(H)}(u)+s_{L(H)}(\mathrm{v})-2\right)}{\left(s_{L(H)}(u) s_{L(H)}(\mathrm{v})\right)}}
$$

where $s_{L(H)}(u)$ is the sum of valency of the nodes adjacent with $u$ and $s_{L(H)}(v)$ is the valency of the nodes adjacent with $v$ of line graph of $H$. 
The difference of geometric and arithmetic mean is defined as the Geometric Arithmetic index [12] introduced by Vukicevic and Furtula and its edge version is given by

${ }_{e} G A(H)=\sum_{u v \in E(L(H))} \frac{2 \sqrt{d_{L(G)}(u) d_{L(G)}(v)}}{d_{L(G)}(u)+d_{L(G)}(v)}$

and its fifth version is defined as

$$
{ }_{e} G A_{5}(H)=\sum_{u v \in E(L(H))} \frac{2 \sqrt{s_{L(H)}(u) s_{L(H)}(v)}}{s_{L(H)}(u)+s_{L(H)}(v)}
$$

where $d_{L(H)}(u)$ is the valency of the node ${ }_{e} d_{L(H)}(v)$ is the valency of the node $v$ of line graph $H$ and $s_{L(H)}(u)$ is the sum of valency of the nodes adjacent with $u s_{L(H)}(v)$ is the valency of the nodes adjacent with $v$ of line graph of $H$.

In [6] G.H.Fath-Tabar et al. reported the PI, Szeged and Zagreb group indices of the Tetrameric 1,3 Adamantane (TA[n]) and Ali $\mathrm{AB}$ et al [1] gave explict formulae for finding Szeged index, Wiener index and edge Wiener index of an infinite array of Tetrameric 1,3 Adamantane (TA[n]). In this article, we compute the edge version topological invariants of an infinite array of Tetrameric 1,3 Adamantane (TA[n]).

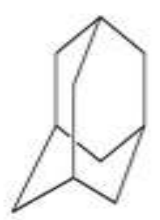

(a)

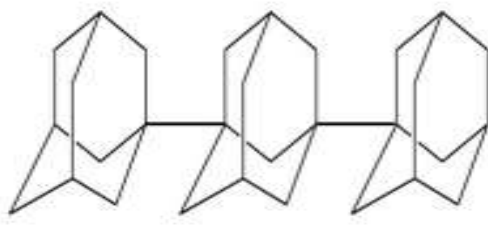

(b)
Fig 1: (a) Tetrameric1,3 adamantane[TA(1)], (b) Tetrameric 1,3 adamantane $[\mathrm{TA}(3)]$

\section{Edge Version Molecular Descriptors of Te- trameric 1, 3 Adamantane [TA (N)]}

Unless stated otherwise, let $M$ be the molecular graph of Tetrameric 1,3 Adamantane (TA[n]) and $G$ be the line graph of the molecular graph of Tetrameric 1,3 Adamantane (TA[n]), $n>2$. There are $13 n-1$ nodes and $24 n-6$ links in $G$. Out of $13 n-1$ nodes $6 n+6$ nodes are of degree three, $(6 n-6)$ nodes of degree four and $(n-1)$ nodes of degree six, $G$ has $24 n-6$ links with degree vertices three, four and six.

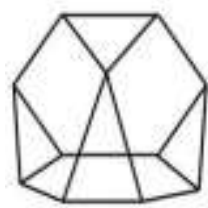

(a)

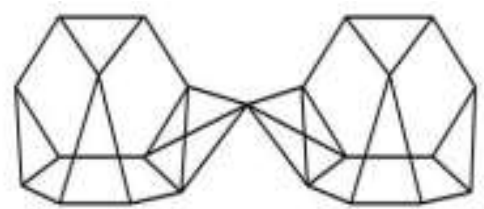

(b)
Fig 2: (a) Line graph of tetrameric 1,3 adamantane L[TA(1)], (b) Line graph of tetrameric 1,3 adamantane $\mathrm{L}[\mathrm{TA}(2)]$

Let us consider the edge partition of $G$ based on degree, the first edge partition has $(7 n+10)$ links with $d_{L(M)}(u)=d_{L(M)}(u)=3$, the second edge partition has $(4 n-2)$ links with $d_{L(M)}(u)=3$ and $d_{L(M)}(u)=4$, the third edge partition has $(7 n-8)$ links with $d_{L(M)}(u)$ $=d_{L(M)}(u)=4$ and the fourth edge partition has $(6 n-6)$ links with $d_{L(M)}(u)=4$ and $d_{L(M)}(u)=6$. We now compute the edge version of Randi'c, Zagreb, Atom Bond Connectivity with its fourth version and Geometric Arithmetic index with its fifth version of $H$.

\section{Theorem 3.1.}

If $G$ be the line graph of molecular graph of Tetrameric 1,3 Adamantane $[\mathrm{TA}(\mathrm{n})]$, then

${ }_{e} R_{\alpha}(G)= \begin{cases}(1 / 3)[7 n+10+(n-2) \sqrt{3}]+(1 / 4)[7 n-8+(2 n-2) \sqrt{6}] & a=-1 / 2 \\ 49 n-2+(8 n-4) \sqrt{3}+(12 n-12) \sqrt{3} & \alpha=1 / 2 \\ 367 n-206 & \alpha=1 \\ (259 / 144) n+(7 / 36) & a=-1\end{cases}$

Proof. Let $G$ be the line graph of molecular graph of Tetrameric 1,3 Adamantane [TA(n)]. Since from (1) we have

$$
e R_{a}(\mathrm{G})=\sum_{w \in \in E(L(\mathrm{M}))}\left(d_{L(\mathrm{M})}(u) d_{L(\mathrm{~N})}(\mathrm{v})\right)^{\alpha}
$$

For $\alpha=-1 / 2$, we have

$$
\begin{aligned}
& e R_{-1 / 2}(\mathrm{G})=\sum_{w \in E(L(\mathrm{M}))}\left(d_{L(\mathrm{M})}(u) d_{L(\mathrm{M})}(\mathrm{v})\right)^{-1 / 2} \\
& =\sum_{(3,3) \in E(L(\mathrm{M}))}(3 \times 3)^{-1 / 2}+\sum_{(3,4) \in E(L(\mathrm{M}))}(3 \times 4)^{-1 / 2}
\end{aligned}
$$

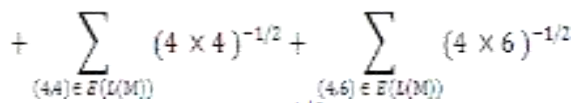

$$
\begin{aligned}
& =(7 n+10)(3 \times 3)^{-1 / 2}+(4 n-2)(3 \times 4)^{-1 / 2} \\
& +(7 n-8)(4 \times 4)^{-1 / 2}+(6 n-2)(4 \times 6)^{-1 / 2}
\end{aligned}
$$

After simplification, we get

$$
\begin{aligned}
& e R_{-1 / 2}(G)=\left(\frac{1}{3}\right)(7 n+10+(n-2) \sqrt{3})+\left(\frac{1}{4}\right)(7 n-8+(2 n-2) \sqrt{6}) \\
& \text { Similarly for } \alpha=1 / 2,1,-1, \text { we get } \\
& e R_{1 / 2}(G)=49 n-2+(8 n-4) \sqrt{3}+(12 n-2) \sqrt{3} \\
& e R_{1}(G)=367 n-206 \\
& e R_{-1}(G)=\left(\frac{259}{144}\right) n+\left(\frac{7}{36}\right) .
\end{aligned}
$$

\section{Theorem 3.2.}

If $G$ be the line graph of molecular graph of Tetrameric 1,3 Adamantane [TA(n)], then $e M_{1}(G)=186 n-78$.

Proof. Let $G$ be the line graph of molecular graph of Tetrameric 1,3 Adamantane [TA(n)]. Since from (2) we have

$$
\begin{aligned}
& e M_{1}(\mathrm{G})=\sum_{w \in E(L(\mathrm{M}))}\left(d_{L(\mathrm{M})}(u)+d_{L(\mathrm{M})}(\mathrm{v})\right) \\
& =\sum_{(3,3) \in E(L(\mathrm{M}))}(3+3)+\sum_{(3,4)}(3+4)
\end{aligned}
$$

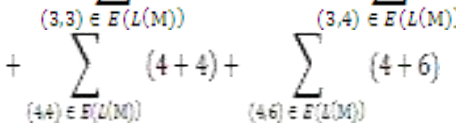

$$
\begin{aligned}
& \text { By using edge partition, we get } \\
& =(7 n+10)(3+3)+(4 n-2)(3+4) \\
& +(7 n-8)(4+4)+(6 n-6)(4+6)
\end{aligned}
$$

After simplification, we get

$e M_{1}(\mathrm{G})=186 n-78$.

\section{Theorem 3.3.}

$\mathrm{f} G$ be the line graph of molecular graph of Tetrameric 1,3 Adamantane $[\mathrm{TA}(\mathrm{n})]$, then

$e A B C(G)=2 \sqrt{3}(n-1)+\left(\frac{14 n+20}{3}\right)+\left(\frac{2 n-1}{3}\right) \sqrt{15}+\left(\frac{7 n-8}{4}\right) \sqrt{6}$ 
Proof.

Let $G$ be the line graph of molecular graph of Tetrameric 1,3 Adamantane[TA(n)]. Since from (3) we have

$$
\begin{gathered}
{ }_{e} A B C(G)=\sum_{w \in \in E\langle L(M))} \sqrt{\frac{d_{L\langle M\rangle}(u)+d_{L(M)}(v)-2}{d_{L\langle M\rangle}(u) d_{L\langle M\rangle}(v)}} \\
{ }_{e} A B C(G)=\sum_{(3,3) \in E(L(M))} \sqrt{\frac{3+3-2}{3 \times 3}}+\sum_{\langle 3,4) \in E(L(M))} \sqrt{\frac{3+4-2}{3 \times 4}} \\
+\sum_{\langle 4,4) \in E(L(M))} \sqrt{\frac{4+4-2}{4 \times 4}}+\sum_{(4,6) \in E(L, M))} \sqrt{\frac{4+6-2}{4 \times 6}}
\end{gathered}
$$

By using edge partition, we get

$$
\begin{aligned}
& \text { e } A B C(G)=(7 n+10) \sqrt{\frac{3+3-2}{3 \times 3}}+(4 n-2) \sqrt{\frac{3+4-2}{3 \times 4}} \\
& \quad+(7 n-8) \sqrt{\frac{4+4-2}{4 \times 4}}+(6 n-6) \sqrt{\frac{4+6-2}{4 \times 6}}
\end{aligned}
$$

After simplification, we get

$$
e A B C(G)=2 \sqrt{3}(n-1)+\left(\frac{14 n+20}{3}\right)+\left(\frac{2 n-1}{3}\right) \sqrt{15}+\left(\frac{7 n-8}{4}\right) \sqrt{6}
$$

\section{Theorem 3.4.}

If $G$ be the line graph of molecular graph of Tetrameric 1,3 Adamantane[TA(n)], then

$e G A(G)=14 n+2+\left(\frac{16 n-8}{7}\right) \sqrt{3}+\left(\frac{12 n-12}{5}\right) \sqrt{6}$.

\section{Proof.}

Let $G$ be the line graph of molecular graph of Tetrameric 1,3 Adamantane $[\mathrm{TA}(\mathrm{n})]$. Since from (5) we have

$$
\begin{aligned}
& e^{G A(G)}=\sum_{u v \in E(L(M))} \frac{2 \sqrt{d_{L(M)}(u) d_{L(M)}(v)}}{d_{L(M)}(u)+d_{L(M)}(v)} \\
& =\sum_{u v \in(3,3)} \frac{2 \sqrt{3 \times 3}}{3+3}+\sum_{u v \in(3,4)} \frac{2 \sqrt{3 \times 4}}{3 \times 4} \\
& +\sum_{u v \in(4,4)} \frac{2 \sqrt{4 \times 4}}{4+4}+\sum_{u v \in(4,6)} \frac{2 \sqrt{4 \times 6}}{4+6}
\end{aligned}
$$

By using edge partition, we get

$$
\begin{gathered}
e_{e} G A(G)=2(7 n+10) \frac{2 \sqrt{3 \times 3}}{3+3}+2(4 n-2) \frac{2 \sqrt{3 \times 4}}{3 \times 4} \\
+2(7 n-8) \frac{2 \sqrt{4 \times 4}}{4+4}+2(6 n-6) \frac{2 \sqrt{4 \times 6}}{4+6}
\end{gathered}
$$

After simplification, we get

$$
e G A(G)=14 n+2+\left(\frac{16 n-8}{7}\right) \sqrt{3}+\left(\frac{12 n-12}{5}\right) \sqrt{6}
$$

Table 1: The Edge Partition of $G$

$\left(s_{G}(u), s_{G}(v)\right) \quad(9,9) \quad(9,10)(10,10)(10,17)(17,17)(17,24)$

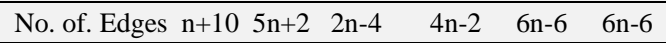

\section{Theorem 3.5}

If $G$ be the line graph of molecular graph of Tetrameric 1,3 Adamantane $[\mathrm{TA}(\mathrm{n})]$, then
$8 A B C_{4}(G)=\left(\frac{4 n+58}{9}\right)+\left(\frac{3 n-6}{5}\right) \sqrt{2}+\left(\frac{5 n+2}{3}\right)\left(\frac{\sqrt{17}}{\sqrt{10}}\right)+(6 n-6)\left(\left(\frac{\sqrt{6}}{17}\right)+\left(\frac{\sqrt{39}}{\sqrt{408}}\right)\right)$

\section{Proof.}

Let $G$ be the line graph of molecular graph of Tetrameric 1,3 Adamantane $[\mathrm{TA}(\mathrm{n})]$. Since from (4) we have

$$
\begin{aligned}
& e A B C_{4}(\mathrm{G})=\sum_{w \in \in E(L(M))} \sqrt{\frac{\left(s_{L(M)}(u)+s_{L(M)}(\mathrm{v})-2\right)}{\left(s_{L(M)}(u) s_{L(M)}(\mathrm{v})\right)}} \\
& =\sum_{w \in \in(9,9)} \sqrt{\frac{9+9-2}{9 \times 9}}+\sum_{u \in \in(9,10)} \sqrt{\frac{9+10-2}{9 \times 10}}+\sum_{u \in \in(10,10)} \sqrt{\frac{10+10-2}{10 \times 10}} \\
& +\sum_{u v \in(10,17)} \sqrt{\frac{10+17-2}{10 \times 17}}+\sum_{u \in \in(17,17)} \sqrt{\frac{17+17-2}{17 \times 17}++}+\sum_{u \in \in(17,24)} \sqrt{\frac{17+24-2}{17 \times 24}} \\
& =(n+10) \sqrt{\frac{9+9-2}{9 \times 9}}+(5 n+2) \sqrt{\frac{9+10-2}{9 \times 10}}+(2 n-4) \sqrt{\frac{10+10-2}{10 \times 10}} \\
& +(4 n-2) \sqrt{\frac{10+17-2}{10 \times 17}}+(6 n-6) \sqrt{\frac{17+17-2}{17 \times 17}}+(6 n-6) \sqrt{\frac{17+24-2}{17 \times 24}}
\end{aligned}
$$

After simplification we get

$\left.e A B C_{4}(G)=\left(\frac{4 n+58}{9}\right)+\left(\frac{3 n-6}{5}\right) \sqrt{2}+\left(\frac{5 n+2}{3}\right)\left(\frac{\sqrt{17}}{\sqrt{10}}\right)+(6 n-6)\left(\frac{\sqrt{6}}{17}\right)+\left(\frac{\sqrt{39}}{\sqrt{408}}\right)\right)$

\section{Theorem 6.}

If $G$ be the line graph of molecular graph of Tetrmeric 1,3 Adamantane $[\mathrm{TA}(\mathrm{n})]$, then

$G G A_{5}(G)=(7 n+4)+\left(\frac{30 n+12}{19}\right) \sqrt{10}+\left(\frac{4 n-8}{27}\right) \sqrt{170}+\left(\frac{12 n-12}{41}\right) \sqrt{408}$

Proof.

Let $G$ be the line graph of molecular graph of Tetrameric 1,3 Adamantane $[\mathrm{TA}(\mathrm{n})]$. Since from (6) we have

$$
\begin{aligned}
& e G A_{5}(\mathrm{G})=\sum_{w \in E(L(\mathrm{M}))} \frac{2 \sqrt{\left(\mathrm{s}_{L(\mathrm{M})}(u) s_{L(M)}(\mathrm{v})\right)}}{\left(\mathrm{s}_{L(\mathrm{M})}(u)+s_{L(\mathrm{w})}(\mathrm{v})\right)} \\
& e_{e} G A(G)=\sum_{\{9, j] \in E(L(M))} \frac{2 \sqrt{9 \times 9}}{9+9}+\sum_{\{9,10) \in E(L(M))} \frac{2 \sqrt{9 \times 10}}{9+10}+\sum_{(10,10) \in E(L(M)]} \frac{2 \sqrt{10 \times 10}}{10+10} \\
& +\sum_{(10,17) \in E(L(M))} \frac{2 \sqrt{10 \times 17}}{10+17}+\sum_{(17,17) \in E(L(M))} \frac{2 \sqrt{17 \times 17}}{17+17}+\sum_{(17,24) \in E(L(M))} \frac{2 \sqrt{17 \times 24}}{17+24}
\end{aligned}
$$

By using edge partition from the Table 1 , we get

$$
\begin{gathered}
=(n+10) \frac{2 \sqrt{9 \times 9}}{9+9}+(5 n+2) \frac{2 \sqrt{9 \times 10}}{9+10}+(2 n-4) \frac{2 \sqrt{10 \times 10}}{10+10} \\
+(4 n-2) \frac{2 \sqrt{10 \times 17}}{10+17}+(6 n-6) \frac{2 \sqrt{17 \times 17}}{17+17}+(6 n-6) \frac{2 \sqrt{17 \times 24}}{17+24}
\end{gathered}
$$

After simplification we get

$$
e G A_{5}(G)=(7 n+4)+\left(\frac{30 n+12}{19}\right) \sqrt{10}+\left(\frac{4 n-8}{27}\right) \sqrt{170}+\left(\frac{12 n-12}{41}\right) \sqrt{408}
$$




\section{Conclusion}

In this article, we computed edge version of topological indices such as Zagreb index, Atom bond connectivity index, Fourth atom bond connectivity index, Geometric Arithmetic index and Fifth Geometric Arithmetic index of Tetrameric 1,3 Adamantane.

\section{References}

[1] Ali AR, Gholam HF \& Far ZG (2012), Some Topological indices of an infinite 1,3 adamantane array, Studia UBB Chemia 57, 151156.

[2] AliMansoori G (2007), Diamondoid molecules, Advances in chemical physics 136, 207-258

[3] AliMansoori G, DeAraujo PLB \& DeAraujo ES (2012), Diamondoid molecules with applications in Biomedicine, World Scientific Publishing Co. Pvt. Ltd, Singapore, pp 279-374.

[4] AnlaiMa (2016), Advancement in application of diamondoids in Organic Geochemistry, Journal of Natural Gas Geoscience 1, 257 265.

[5] Estrada E, Torres L, Rodrigue L \& Gutman I (1998), An atom bond connectivity index : modeling the enthalphy of formation of alkanes, Indian Journal of chemistry 48, 849-855.

[6] FathTabar GH, Azd A \& Elahinezhad N (2010), Some topological indices of tetrameric 1,3 adamantane , Iranian Journal of Mathematical Chemisrty 1, 111-118

[7] Gutman I \& Trinajstic N (1972), Graph Theory and molecular orbitals, total $\pi$-electeon energy of alternate hydrocarbons , Chemical Physical Letters 17, 535-538.

[8] Li X \& Zhao H (2000), Trees with first three smallest and largest generalized Topological indices, MATCH communications in mathematical and in computer chemistry 50, 57-62.

[9] Nadeem MF, Zafar S \& Zahid Z (2016), On Topological Properties of line graphs of certain nanostructures, Applied Mathematics and Computation 273, 125-130.

[10] Randic M (1975), On Charaterisation of molecular branching, Journal of American Chemical Society, 97, 6609-6615.

[11] Schoell M \& Carlson RMK (1999), Diamondoids and oil are not forever, Nature 399, 15-16.

[12] Vukicevic D \& Furtula B (2009), Topological index based on the ratios of geometrical and arithmetical means of end-vertex degrees of edges, Journal of Mathematical Chemistry 46, 1369-1376.

[13] Wiener H (1947), Structural determination of paraffin boling points, Journal of American Chemical Society 69, 17-20. 